\title{
In Vitro
}

National Cancer Institute

\section{Source}

National Cancer Institute. In Vitro. NCI Thesaurus. Code C15263.

In the laboratory; literally, "in glass". 